\title{
The treatment of dysfunctional uterine bleeding with the Mirena IUS: a survival analysis
}

Received: 13 April 2005 / Accepted: 14 July 2005 / Published online: 17 August 2005

(C) Springer-Verlag Berlin / Heidelberg 2005

\begin{abstract}
A questionnaire was sent to 124 women with dysfunctional uterine bleeding (DUB) who had been treated with the Mirena IUS. Prior to treatment, the women had received advice about the side effects of the Mirena IUS. There was a $71 \%$ response rate of which $21 \%$ had the Mirena IUS removed prematurely. The main reasons for removal were pelvic pain and irregular vaginal bleeding. The projected Kaplan-Meier survival rate for the device over the 5-year therapeutic lifetime was $55 \%$. The amenorrhoea rate was $38 \%$ and the oligomenorrhoea rate was $72 \%$. The Mirena IUCD is an effective form of treatment for DUB and the majority of women are likely to continue with this treatment at 5 years.
\end{abstract}

Keywords Mirena IUS - Dysfunctional uterine bleeding

\section{Introduction}

The levonorgestrel-containing intra-uterine contraceptive system (Mirena IUS) is used to treat women with dysfunctional uterine bleeding (DUB). The reported oligomenorrhoea rates range from $75 \%$ to $91 \%$ [1, 2], and the amenorrhoea rate in a randomised controlled trial was $36 \%$ [3]. However, the device may have to be removed prematurely for a variety of reasons in as many as $57 \%$ of users [4]. This may be due to progestogenic side effects, such as weight gain, greasy skin, acne and breast tenderness, and women may also experience pelvic pain and irregular vaginal bleeding. Previous

K. Mukherjee $\cdot$ K. Jones $(\square)$

Department of Obstetrician and Gynaecologist,

The Great Western Hospital, Swindon, Wilts, SN3 6BB, UK

E-mail: Kevin.jones@smnhst.swest.nhs.uk

Tel.: +44-179-3604020 studies have reported that most treatment failures requiring removal of the device occur during the initial 3-6 months [5]. In order to determine the proportion of women who have the device removed over time, a survival analysis on a cohort of patients who had the Mirena IUS fitted to treat DUB was carried out. The indications for removal of the device and the patient's menstrual status were also quantified.

\section{Methods}

One hundred and twenty-four women with a history of menorrhagia without intra-uterine pathology were recruited for the study at a district general hospital. Patients were eligible if they had a uterine cavity (excluding the cervical length) measuring between 6 and $10 \mathrm{~cm}$ in length at hysteroscopy or on trans-vaginal ultrasound scan. The patients were aged between 18 and 55 (mean 41) years. They were excluded if there was intra-uterine pathology or endometrial and cervical abnormalities on histological and cytological examination. Intra-mural and extra-uterine pelvic disease were considered a relative contraindication. Histories of previous caesarean section(s) or clotting abnormalities were not considered a contraindication.

All 124 patients had a Mirena IUS fitted to treat DUB in a "one stop" clinic for abnormal uterine bleeding. They had received both verbal advice and patient information leaflets about the side effects of the Mirena IUS prior to device insertion. A postal questionnaire was sent to all the women. The date of insertion of the device and the patient's age was recorded. The patients were asked if they were still using the device. If it had been removed, they specified the time after insertion this had occurred and the reason for removal. If the device had not been removed, the patients were asked to specify whether they still had periods. Those women who still had a menstrual bleed (more than spotting) were asked to specify whether it was lighter, 
heavier or unchanged compared with their periods before the device was inserted.

\section{Statistical analysis}

Data for analysis was recorded on Microsoft EXCEL software (V.5 for Windows). The survival analysis was undertaken in SPSS version 12.0. Survival probabilities by Kaplan-Meier method for censored data were plotted.

\section{Results}

A cohort of 124 women were sent a questionnaire. There was a $71 \%(88 / 124)$ response rate. Of these patients, $21 \%(18 / 88)$ had the Mirena IUS removed prematurely. The reasons for removal were pelvic pain $50 \%(9 / 18)$, irregular vaginal bleeding 50\% (9/18), and 38\% (7/18) women had the device removed for another reason (weight gain, skin changes, and breast tenderness). The survival probabilities by Kaplan-Meier method for censored data is shown in Fig. 1. At 6 months following insertion of the device, $10 \%$ had been removed $(90 \%$ survival). This increased to $20 \%$ of the devices $(80 \%$ survival rate) at 12 months following insertion. At 36 months, $30 \%$ of the devices had been removed $(70 \%$ survival rate). The projected survival rate of the device over the 5-year therapeutic lifetime of the device was $55 \%$. In those women who still had a Mirena IUS, the amenorrhoea rate was $38 \%(33 / 88)$ and the oligomenorrhoea rate was $72 \%(63 / 88)$.

\section{Discussion}

DUB is defined as the complaint of heavy, prolonged or irregular menstrual bleeding over several consecutive menstrual cycles in the absence of pathology. DUB has a massive impact on women's lives. One in 20 women aged 30-49 years consult their general practitioners each year with this complaint because it has become professionally and socially debilitating. Once referred to a gynaecologist, $60 \%$ of these women will have a hysterectomy within 5 years, and half of all women who have a hysterectomy for this reason have a normal uterus removed [6]. In response to this, the Royal College of Obstetricians and Gynaecologists (RCOG) has developed guidelines for the management of this common condition. The guidelines state that drugs and the Mirena IUS should be offered as first-line treatment options and that surgical interventions such as endometrial ablation or hysterectomy should be offered as second-line therapy [6].

The Mirena IUS consists of a T-shaped intrauterine device sheathed with a reservoir of levonorgestrel in a polydimethylsiloxane cover. The levonorgestrel is released at the rate of $20 \mu \mathrm{g}$ per day for 5 years. The device can be fitted in the outpatient clinic without anaesthetic and exerts its clinical effect by preventing endometrial proliferation and consequently reduces both the duration of bleeding and the amount of menstrual loss. These beneficial effects are due to the slow release of levonorgestrel, which also has unwanted progestogenic side effects necessitating removal of the device prematurely. Our study has shown that overall, $21 \%$ of patients had the Mirena IUS removed prematurely during a 3-year period. The Kaplin-Meier plot (Fig. 1)
Fig. 1 Survival analysis (Kaplan-Meier plot for censored data). The cumulative survival (removal of the Mirena IUS) over time.

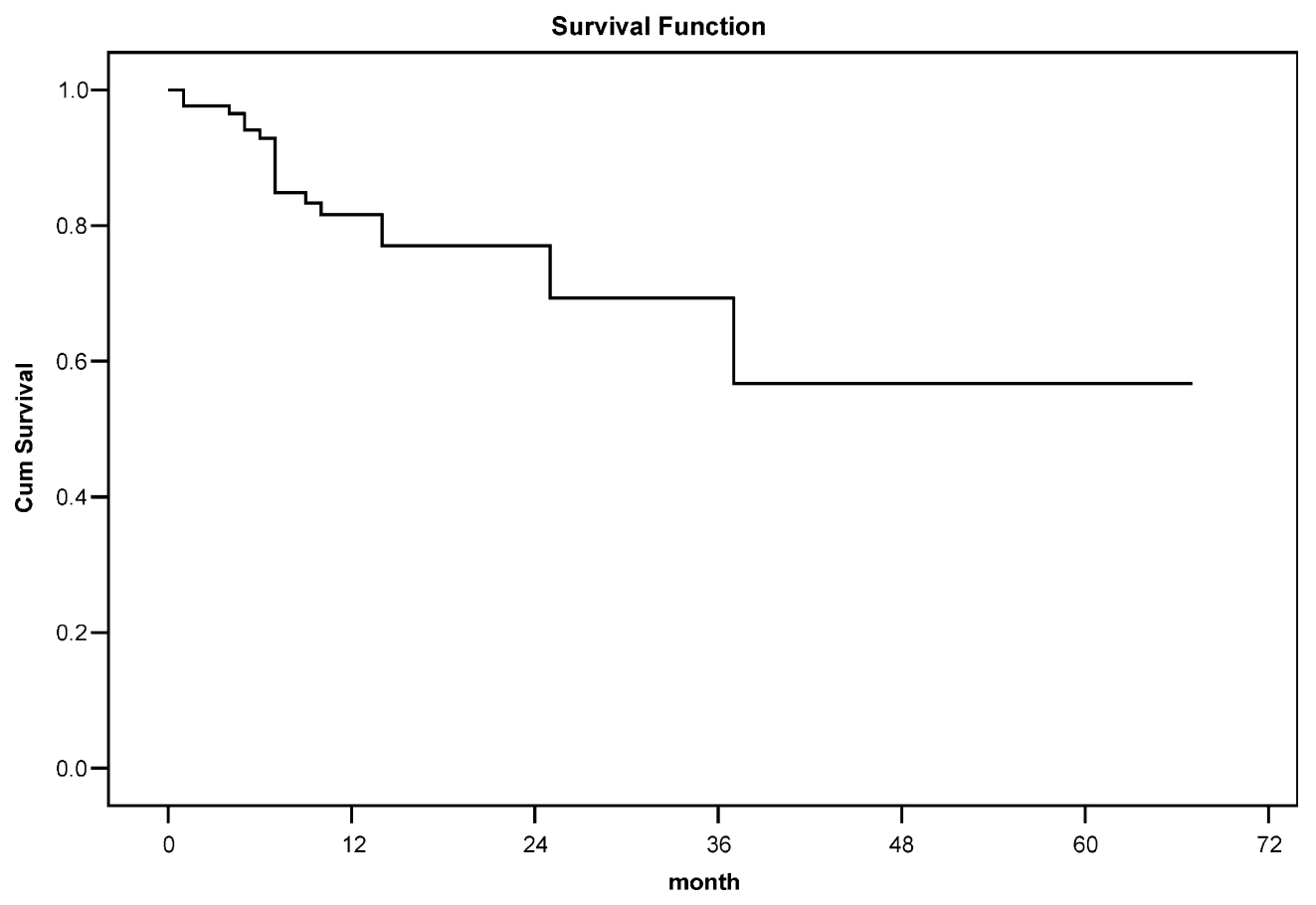


has demonstrated that there was a $10 \%$ failure of treatment (device removal) over the initial 6 months (90\% survival rate). This is a considerably lower initial failure rate than previously reported and it is at variance with the advice given in the product information leaflet. In a study comparing the Mirena IUS to trans-cervical resection of the endometrium (TCRE), $50 \%$ of treatment failures occurred in the initial 6 months after insertion [3] whereas a study comparing the Mirena IUS to hysterectomy found that $58 \%$ of treatment failures occurred in the initial 6 months [4] and in a prospective cohort study, the failure rate was $65 \%$ [5].

Success or failure of treatment with the Mirena IUS is multi-factorial and difficult to predict in an individual case. Studies that have used health-related quality-of-life measures (HRQL) have shown that a low base-line score in HRQL predicts poorer continuation rates with the Mirena IUS [1]. This suggests that women who experience device failures have a lower tolerance of adverse effects because of psychosocial problems. However, the low initial failure rate may also reflect the fact that patients are being encouraged to persist with the Mirena IUS in spite of the known side effects.

Progestogenic side effects have been reported when the Mirena IUS has been used as a contraceptive, and proper counselling of women regarding these symptoms at the time of insertion of the system is provided to improve the continuation rate. In a study by Nagrani et al. [2], progestogenic side effects were reported by $61 \%$ of women but still, their satisfaction with treatment was more than 8 on the visual analogue scale. Effective patient information leaflets and the counselling, which the patients receive in the one stop abnormal bleeding clinic may aid this.

High rates of device expulsion immediately after insertion have been reported [5]. This was not demonstrated in our study. The timing of insertion of the Mirena IUS during the menstrual cycle was not recorded; however, as no devices were removed or expelled within one menstrual cycle, this observation is not directly relevant to the study outcome measure. The overall proportion of women who eventually have the device removed is similar to other studies [2]. The most commonly cited reasons for removal are presented in
Table 1. The main indications were an unacceptable bleeding pattern, pelvic pain, and other causes such as weight gain, skin changes, and personal choice. The projected survival rate of the device over its therapeutic life time (5 years) was $55 \%$. This is a higher rate compared with long-term follow-up studies, which have reported that $49 \%$ [1] and $50 \%$ [2] of women continue to use the device at 5 years.

Various outcome measures have been reported to indicate successful treatment with the Mirena IUS, such as the amenorrhoea rate, which varies widely from $36 \%$ [3] to $75 \%$ [1], and avoidance of surgery for menorrhagia: $67.4 \%$ [2] to $64.3 \%$ [4]. In those women who still had a Mirena IUS in our study, the amenorrhoea rate was $38 \%$ and the oligomenorrhoea rate was $72 \%$, which compares favourably with previous studies. It should be noted, however, that this study has not used an objective measure of menstrual status. Neither was the hormonal status of the women in the study documented. These limitations are important if the efficacy of the Mirena IUS is being compared with other forms of treatment for DUB such as second-generation endometrial ablation devices. However, the objective of this study was to document the premature removal of the device over time, so a quantitative estimate of blood loss and hormonal status were not required. If a patient had little or no change in their menstrual blood loss but wished to continue using the device, this was a taken as a success because the women did not have the Mirena IUS removed.

A study comparing the costs of medical and surgical treatments of menorrhagia [1] has shown that treating menorrhagia with hysterectomy rather than with the Mirena IUS was three times more expensive, and after 5 years of treatment with the Mirena IUS, it was still $40 \%$ less expensive than hysterectomy. This study has demonstrated that early device failure can be prevented, which ought to encourage general practitioners and gynaecologists to recommend this form of treatment for DUB, which will clearly have a positive impact on costs. It has been suggested that introduction of endometrial ablation has increased the overall rate of expensive surgical procedures. In the UK, hysterectomy rates have increased despite the growing

Table 1 Indications for premature removal of the Mirena IUS

\begin{tabular}{llll}
\hline References & Unacceptable bleeding & Pelvic pain & Other symptoms \\
\hline This study & $50 \%(9 / 18)$ & $50 \%(9 / 18)$ & $39 \%$ \\
Hurskainen et al. [1] & $70 \%(42 / 60)$ & $10 \%(6 / 60)$ & $33 \% \mathrm{o}^{\mathrm{a}}$ \\
Nagrani et al. [2] & $48 \%(11 / 23)$ & $39 \%(9 / 23)$ & $35 \% \mathrm{o}^{\mathrm{b}}$ \\
Kittelsen and Istre [3] & $50 \%(3 / 6)$ & $33 \%(2 / 6)$ & $17 \%{ }^{\mathrm{c}}$ \\
Lahteenmaki et al. [4] & $58 \%(7 / 12)$ & $25 \%(3 / 12)$ & $33 \% \mathrm{o}^{\mathrm{d}}$ \\
\hline
\end{tabular}

\footnotetext{
${ }^{\text {a }}$ Progestogenic symptoms including depression

${ }^{\mathrm{b}}$ Other reasons, but not releated to progestogenic side effects

${ }^{\mathrm{c}}$ Skin changes

${ }^{\mathrm{d}}$ Progestogenic symptoms and personal reasons

(Some women cited more than one side effect as the indication for removal)
} 
popularity of endometrial ablation [1]. In Finland, the use of endometrial ablation is low but the Mirena IUS is widely accepted. The national hysterectomy rate in Finland has declined by $13 \%$ since 1998, suggesting that the use of the Mirena IUS is already changing clinical practice. By applying the RCOG guidelines [6], similar changes may occur in the UK, particularly if early device failures can be prevented by counselling and patient information leaflets.

\section{Conclusions}

Our study has demonstrated that early failures of the Mirena IUS in the treatment of DUB can be prevented and that the majority of women are likely to continue with this treatment at 5 years.

Acknowledgements The authors would like to thank Dr Gordon Taylor, University of Bath, UK, for his advice regarding the statistical analysis of the data. Conflicts of interest: None.

\section{References}

1. Hurskainen R, Teperi J, Rissanen P, et al (2004) Clinical outcomes and costs with the levonorgestrel-releasing intrauterine system or hysterectomy for treatment of menorrhagia Randomized trial 5-year follow-up. JAMA 291:1456-1463

2. Nagrani R, Bowen-Simpkins P, Barrington JW (2002) Can the levonorgestrel intrauterine system replace surgical treatment for the management of menorrhagia?. BJOG 109:345-347

3. Kittelsen N, Istre O (1998) A randomized study comparing levonorgestrel intrauterine system (LNG IUS) and transcervical resection of the endometrium (TCRE) in the treatment of menorrhagia. Gynae Endosc 7:61-65

4. Lahteenmaki P, Haukkamaa M, Puolakka J, Riikonen U, et al (1998) Open randomised study of use of levonorgestrel releasing intrauterine system as alternative to hysterectomy. BMJ 316:1122-1126

5. Hidalgo M, Bahamondes L, Perrotti M, Diaz J, et al (2002) Bleeding patterns and clinical performance of the levonorgestrelreleasing intrauterine system (Mirena) up to two years. Contraception 65:129-132

6. RCOG Press (1999) The management of menorrhagia in secondary care. Evidence-based clinical guideline No. 5; http:// www.rcog.org.uk/guidelines/menorrhagiasc.html 